\title{
AN EXPANDED APPROACH TO URBAN RESILIENCE: THE CASE OF ACCRA, GHANA
}

\author{
Luiz Felipe Ribeiro Barbalat Queiroz and Marie Anne Macadar \\ COPPEAD/The Federal University of Rio de Janeiro \\ Rua Pascoal Lemme, 355, Cidade Universitária, Rio de Janeiro, 21941-918, Brazil
}

\begin{abstract}
Urban resilience refers to "the ability of a [urban] system, entity, community or person to adapt to a variety of change conditions and to withstand shocks while still maintaining its essential functions" (World Bank Group, 2015). This ability to adapt and change in response to shocks and stressors becomes increasingly important in an urban context. We can consider three significant reasons for this situation: (1) increased urbanization often leads to poorly-managed growth and unsustainable land use; (2) rapid urbanization is associated with an increased vulnerability to environmental and social shocks and stressors; and (3) urban poor populations are more likely to be impacted by these shocks and stressors. When urbanization is carried out in an inclusive and resilient manner, it enables the sustained reduction of poverty and improvements in shared prosperity. The main objective of this paper is presenting a case study argument about the efficacy and deficiency of some city policies, as well as propose recommendations on how technology can improve the city infrastructure and build resilient systems to withstand shocks and stressors in Accra. For this study, an expanded literature review on urban resilience, ICT technologies, and smart cities practices was conducted. Data analysis, mapping techniques, and field trip images complete the analysis of the case study. As part of the data collection strategy, interviews were conducted, from 2017 to 2018, with officials from the national and local governments, ministries, and World Bank Group staff in Accra, Ghana.
\end{abstract}

\section{KEYWORDS}

Accra, Ghana, Case Study, World Bank Group, Urban Resilience, Technology, ICTs, Smart Cities, Sustainable Development

\section{INTRODUCTION}

As defined by the World Bank Group (WBG) in its paper, "An Expanded Approach to Urban Resilience," urban resilience refers to "the ability of an [urban] system, entity or community or person to adapt to a variety of changing conditions and to withstand shocks while still maintaining its essential functions". This ability to adapt and change in response to shocks and stressors becomes increasingly important in an urban context for three major reasons: (1) rapid urbanization often leads to poorly-managed growth and unsustainable land use; (2) rapid urbanization is associated with an increased vulnerability to environmental and social shocks and stressors; and (3) urban poor populations are more likely to be impacted by these shocks and stressors. When urbanization is carried out in an inclusive and resilient manner, it enables the sustained reduction of poverty and improvements in shared prosperity.

Given this broad new perspective of building sustainable urbanization through urban resilience, the IEG plans to carry out "Towards Urban Resilience: An Evaluation of the World Bank Group's Support (2007-2017)", a major evaluation of the WBG's Urban Resilience Operations as it seeks to scale up its advice and investment in this domain. The objective of this evaluation is to assess how well the WBG is helping clients to strengthen resilience mechanisms - to cope, recover, adapt and transform - in the face of acute shocks and chronic stresses, and it focuses on how these initiatives are linked to the WBG's broader poverty reduction goals. This evaluation also includes the preparation of case studies to both collect data on the main attributes of urban resilience and to map the contribution of key stakeholders in specific cities. 
This article will present the preliminary findings of the IEG's Accra, Ghana case study conducted through field interviews during 2017 and 2018. It is intended to objectively evaluate the activities of the WBG in the region, generate lessons from experience, provide accountability to shareholders, and share evaluative evidence to help the organization deliver better services and results to its clients. This is a preliminary case study for the IEG team to use as a foundation for their deeper analysis of the WBG's portfolio in Accra. The IEG's fieldwork in Accra was framed around exploring the legacy of the WBG's City Strength Diagnostic in the city, a five-step program that aimed to engage various stakeholders across the Greater Accra Metropolitan Area (GAMA). The diagnostic aimed to identify shocks and stresses impacting the region and to prioritize relevant action items. This article will outline findings related to the City Strength Diagnostic and will also explore the following projects in the area: Land Use Project; the EDGE program; Local Government Capacity Support Project; Water and Sanitation Project; and the Transport Project. Lastly, this memo will focus on providing a snapshot of the transport, water and sanitation, land use, local government, and private sectors' reflections on the WBG's contribution to the resilience narrative in Accra today.

\section{METHODOLOGY}

For the purpose of this case study, the authors carried out both primary and secondary research in relation to the WBG portfolio, with preliminary research involving a review of various WBG projects in the GAMA Region. The authors narrowed its focus to WBG projects falling in the following four domains: transport, water and sanitation, land-use and planning, and local government. As part of the data collection strategy, interviews were conducted with officials from the national and local governments, ministries, and WBG staff in Accra. Interviews were structured around two sets of qualitative questions developed by the IEG staff. The first set aimed to gather information on how and how well WBG activity has contributed to the development of resilience characteristics in regard to designing WBG projects, generating client awareness, and enhancing client coping, adapting, and transforming capabilities in the face of shocks and stressors. The second set aimed to gather information on how and to what extent the WBG has leveraged its knowledge, human capital and financial resources to help clients achieve urban resilience. For the second set of questions, relevant interview data included information on different partnership arrangements that were part of these efforts, as well as cross-sectoral collaboration across the public and private sector.

\section{CITY STRENGTH}

Interviewees reported that the City Strength Diagnostic has been a prominent force in moving resilience efforts forward in Accra. According to City Strength consultant, Rachael Dede Annan, sectoral teams have bought into the City Strength approach, and awareness is being packaged into projects across sectors, despite there being few mechanisms in place to evaluate the City Strength approach. However, throughout the interviews, it was not inherently clear that all stakeholders held the same positive regard for the City Strength process.

Some interviewees felt significant ownership over the resulting document, including Adenta Municipal Planning Director, Akwasi Owusu-Afriyie, who participated in the workshops which formed it, and felt the approach and accompanying terminology had come from the people directly involved in the process. Conversely, Accra Metropolitan Planning Director, Kwadwo Yeboah, noted that the primary problem related to the City Strength Diagnostic is the lack of implementation occurring around initiatives birthed by the exercise. Additionally, the Deputy Director of Policy, Planning, and Budget for the Ministry of Environment, Science, Technology, and Innovation (METSI), Lydia Essuah, reported lack of coordination as a major obstacle in implementing the City Strength recommendations. This has been in part due to the fact that individual ministries have their own measures and procedures for monitoring programs, and thus, do not have a coordinated approach with which to move forward. Lydia also reported that METSI was approached to take on a coordinating role following the City Strength Diagnostic.

Overall, there is a general consensus that the City Strength document has aided government entities and stakeholders in better identifying shocks, stresses, and methods for coping, adapting, and transforming. The City Strength process brought a host of stakeholders together from across sectors to better plan resilience measures, and increased awareness throughout government bodies, which has started to filter through to 
constituents. Kwadwo Yeboah posited that the City Strength document has provided the government a concrete outline with which to gain financing, and has brought previously-overlooked spatial planning issues to light, while Anthony Mompi, Head of the LEKMA Physical Planning Department, reported that it allowed he and his colleagues to begin viewing related problems through a "resilience lens." Finally, Akwasi Owusu-Afriyie also reported that the City Strength Diagnostic motivated the establishment of new communication channels across government bodies, with workshop participants creating large WhatsApp and Telegram groups to share knowledge and information with one another.

\section{SECTORIAL ISSUES}

\subsection{Transportation}

According to research conducted by the World Bank, road maintenance is the most important resilience change for the transport sector in Accra (Smith, 2015). This challenge is exacerbated by flood risks and the fact that roads are not routinely managed. Following the 2015 flood, the Transport Program restructured its financing, and reallocated 83 million dollars to institute a coping program focused on drainage creation. Other coping efforts undertaken by the transport sector include road maintenance and updating the structure of the previously steel Nafladzo River Bridge, in order to increase inclusivity and reach the community across the river that was cut off during flooding. In terms of adaptation, and physically altering road design in light of these risks, local government authorities listed cost, culture, and inherent restrictions in urban design (i.e. the impact of the changes on urban movement) as obstacles (African Development Bank, 2016).

\subsection{Land Use \& Planning}

Ghana is heavily dependent on primarily land-based products for its growth and socio-economic development, including agriculture, mining, and timber. In both rural and urban areas of Accra, growing population, the diminishing supply of land, inter and intra-regional migration, and urbanization, have strongly contributed to a growing demand for land throughout the country. The unmet demand has resulted in inflationary pressure in the prices of land and property, and has created uncertainty and a lack of transparency in the land delivery system (Agyarko, n.d.).

According to the Food and Agriculture Organization of the United Nations, the failure of public land agencies to develop and implement policies to improve the land management and administration systems has undermined national and international investor confidence in the land and other sectors of the economy. In addition, Accra is experiencing urban sprawl and undertaking transformative strategies that require holistic change. The urban metropolitan areas of Accra are facing difficulty managing population growth, which is exacerbated by a highly-competitive job market, and a high cost of living. These problems can drive local residents, mostly youth, to migrate to either rural or small towns located on the outskirts of the city. These small towns are situated in a tropical region with soil rich in minerals and adequate for agriculture. There are potential opportunities for local governments to improve the linkage between rural and urban regions. However, this strategy can also exacerbate urban sprawl (Addae-Bosompra, 2009).

\subsection{Public-Private Partnerships}

To foster expanded inclusion of the private sector, the IFC launched the EDGE (Excellence in Design for Greater Efficiencies) program in Accra in May 2017. The program focuses on engaging government, developers, and homeowners to think about resource-efficient building designs. There is lacking information on how to build "green" and the possible benefits that accompany this course. This process can be seen as unmanageable and costly, with few government and financial incentives in place pressing private actors to comply. In addition, the program is responsible for the creation of free, publicly-accessible software, which shows developers how to design green and the advantages of doing so from a cost perspective. 
The software is user-friendly, and geared toward smaller developments, but provides actionable recommendations for improving green design. The purpose of the software (EDGE software, n.d) is to show developers that going green is not only environmentally responsible, but a sound business decision as well. In scaling up, the program has recently entered a working relationship with the EPA, which is involved in all large projects in Accra, and is actively pressuring developers to comply with EDGE standards. EDGE has simultaneously focused on engaging with various government stakeholders to improve and solidify Ghanaian building codes, the final versions of which are currently under review before finalization. Lastly, the IFC has begun working closely with financial institutions to incentivize financing for projects interested in green design.

\subsection{Water \& Sanitation}

The secretary of Water \& Sanitation in Accra has been especially instrumental in improving water and sanitation issues since the 2015 floods, which took away civilians' lives, and brought to light the issue of poor sanitation and environmental habits, as well as inadequate drainage facilities in Accra. According to different online newspapers, there is a significant number of Accra's population that do not have access to good quality water, mostly in low-income areas. Similar obstacles have been faced in integrating robustness into the sector's resilience efforts due to lack of data (Water \& Sanitation for the Urban Poor, 2018).

According to reports released by the World Bank Group (GH-GAMA Sanitation and Water Project, 2019), Accra does not have a centralized GIS database available for local reference, and each government agency reported having its own data. Moreover, interviewees from the WASH Project Coordination Unit stated that the sanitation data that is available is outdated. As an example, they mentioned that the data being used in the region is almost 15 years old and still categorizes Accra as one of the dirtiest cities in the world. In reality, this is no longer the case.

The lack of a centralized database makes it much more difficult to report and collect data on informal activities, such as having a toilet in-home. UNICEF and the WBG have been working to collect data in Accra, and the WBG has also provided support to the government in obtaining data and highlighting the important issues the data revealed. Although Accra does not currently have an asset management system in place for the drainage infrastructure, there is an intention to create a master plan to establish this system (Project Information Document (PID) Concept Stage, 2015).

\section{RECOMMENDATIONS}

\subsection{Increase Awareness at Different Levels}

There is a need to increase awareness and access to the existing asset management system, so that different stakeholders can keep track of projects and existing infrastructure. In addition, there is also a need to create awareness and provide training to qualify community leaders to advocate for urban resilience and educate residents to become environmentally friendly. For this, the radio and social media platforms have been used as tools for advocacy and community engagement. The importance of the radio in raising public awareness around resilience themes and providing a platform for local Ghanaians to air their grievances, holding public officials accountable is emphasized by the Ghana Community Radio Network Website. Some community leaders reported to the radio channel a remaining need for raising awareness and for collaboration, referring to examples such as the Transport Sector Working Group, which brings together donors and government. However, collaboration was also reported to be difficult due to diverging opinions on priorities and solutions (Ghana Community Radio Network, n.d.).

\subsection{Increase Public-Private Partnerships}

It is imperative to increase public-private partnerships to capture more funds for smart urban infrastructure. In order to overcome capacity and funding hurdles, there is a need for private companies and international organizations to assist in combating stresses and shocks to move towards adaptive and transformative 
mechanisms. In addition, private companies can also assist local government authorities with the necessary knowledge to build mapping platforms, because there is a need to have a GIS system integrated with formal databases. This includes the sharing of knowledge products and common databases for collaboration and coordination of resilience efforts. The implementation of open data platforms increases transparency and decreases corruption. An example of how public-private partnerships can help the city authorities finance smart infrastructures is the creation of a control center for traffic in Accra. This control center enables the Department of Urban Roads to monitor 165 traffic lights in Accra (Traffic signal system to be centrally controlled, 2013).

\subsection{Solve Social \& Economic Problems Caused by Urban Sprawl}

According to William Frimpong-Bonsu (2002), "The city of Accra has grown dramatically over the years from a tiny settlement into a metropolitan standard called the Greater Accra Metropolitan Area (GAMA) comprising the districts of Accra, Tema and Gama with the emergence of some new residential settlements and the incorporation of old villages and settlements at the peripheral zone. The three districts have become physically and functionally one single urbanized area and it is the largest urban agglomeration in the country." Residents of suburbia and local government authorities are worried that this process, coupled with patterns of urban sprawl, could cause an increase in traffic congestion and air pollution; reduce open and green spaces; destroy farmlands; increase spatial segregation; and impose additional costs of living on neighboring cities.

\subsection{Increase Levels of Coordination Among Different Agencies}

When there is coordination between city systems and agencies means that knowledge is shared, planning is collaborative and strategic, and decision-making is based on investments that are mutually supportive towards a common outcome. Exchange of information between systems enables them to function collectively and respond rapidly through feedback loops occurring throughout the city. Moreover, this lack of coordination regularly leads to misalignment of tasks, wasted efforts, and disagreements between government bodies. For example, the Department of Urban Roads is responsible for drains in urban areas, while Hydrological Services monitors primary drains. These primary drains cut across municipalities. Thus, the municipalities are also responsible, and coordination becomes an obstacle. In order to solve this issue, the national government should create and implement an online platform where different agencies can simultaneously connect and share information and knowledge among each other (Dahiya et al., 2010).

\subsection{Ensure City Resilience to Extreme Weather Events}

The 2015 flooding impacted various areas of the city, mainly low-income neighborhoods where most of the drains were obstructed with garbage. Because of that, the Ghanaian Federal Agency for Disaster Mitigation and Response launched an initiative that sought to rebuild affected infrastructure, and promote resilience of existing and future developments. This core principle is a fundamental policy to improve residents' quality of living, as well as to increase property values throughout the city.

\subsubsection{Strategy 1: Protect Waterfront from Flooding}

- Provide retractable storm surge barriers at the bulkhead of rivers and cliffs;

- Install sirens and audio alert systems around the most vulnerable flooding areas to inform residents about evacuation procedures when needed; and

- Educate local residents on how to act during a dangerous situation provoked by a storm.

\subsubsection{Strategy 2: Improve Resilience of Existing Infrastructure}

- Shift critical building infrastructures that are located under sea level to above flooding levels;

- Inspect inhabited buildings after occurrence of intense rains, because enclosed water bodies can attract mosquitos, including the ones that are responsible to carry both Dengue and Zika viruses;

- City agencies should prioritize responses to chronic stresses over acute shocks; and

- Promote partnerships between universities and local government agencies to encourage and educate students to become community leaders and act as first responders when an extreme event occurs. 


\section{CONCLUSION}

The case study provided strategies on how technology and city policies can promote urban resilience and socioeconomic development in Accra, Ghana. For this, it is imperative that different stakeholders continue to engage in developing efforts to track and solve the problems associated with urban resilience in the city. An expanded literature review and different news articles revealed flooding as one of the major and chronic stresses on the city, with significant implications on areas from drains to disease outbreak. During the article, it was noticeable that both national and local government authorities were working towards solving the main stressors and shocks that Accra faces today, however, there was still a need for better coordination and cooperation among these two levels of government. It is important to mention that this was a preliminary study that needs in-depth research and analysis of the data collected in the field to provide better oriented decision-making techniques and recommendations, nonetheless a list of key findings is stated below.

- Stakeholders in Accra tend to focus on coping mechanisms in response to shocks and stressors. Additionally, WASH interview participants noted that their group prioritizes responses to chronic stresses over acute shocks;

- There is a lack of capacity and funding to pursue adaptive and transformative strategies;

- Flooding is a major chronic stress with implications on sanitation practices, cholera incidence, waste dumping, and defecation habits;

- The City Strength Diagnostic has been a useful guide in generating awareness throughout different levels of Ghanaian society. However, there is a lack of capacity surrounding implementation of resilience measures proposed in the City Strength document;

- There is a lack of significant coordination and cooperation among certain stakeholders in Accra, particularly related to data-sharing within GIS and asset management systems; and

- There are instances of positive private sector participation in resilience efforts, but further alignment of goals between public and private entities is needed.

\section{ACKNOWLEDGEMENT}

We thank all the governmental authorities and local community larders who shared their experiences and information with us during the interview process conducted to collect data for this case study.

\section{REFERENCES}

Agyarko, T. (n.d.). Forestry Outlook Study for Africa (FOSA). Retrieved December 8, 2019, from http://www.fao.org/tempref/docrep/fao/003/AB567E/AB567E00.pdf

Excellence in Design for Greater Efficiencies. (n.d.). Retrieved December 8, 2019, from https://www.edgebuildings.com/.

EDGE software (n.d.). Excellence in Design for Greater Efficiencies. Retrieved December 11, 2019, from https://www.edgebuildings.com/software/

Project Information Document (PID) Concept Stage. (2015, September 8). Retrieved December 7, 2019, from http://documents.worldbank.org/curated/en/345511468037601849/pdf/PID-Print-P151026-09-08-20151441752043616.pdf

Dahiya, Bharat \& Andersson, Cecilia \& Auclair, Christine \& Barth, Bernhard \& Buhren, Karin \& Candiracci, Sara \& Dercon, Bruno \& Gachocho, Mary \& Galezia, Krystyna \& Hossain, Maharufa \& Kadhim, Muhammad \& Kishiue, Akiko \& Materu, Jossy \& Muhoro, Teckla \& Mwaura, John \& Njenga, Cecilia \& Sommer, Kerstin \& Miller, Edward \& Petrella, Laura \& Sietchiping, Remy. (2010). Planning Sustainable Cities: UN-HABITAT Practices and Perspectives.

Ghana Community Radio Network. (n.d.). Retrieved November 19, 2019, from http://gcrn.org.gh/

Frimpong-Bonsu, W. (2002, September 15). Ghana: Sprawl in the Greater Accra Metropolitan Area. Retrieved November 22, 2019, from https://allafrica.com/stories/200209161062.htm.

Addae-Bosompra, E. (2009, October 13). Urban Sprawl: A New Epidemic In Accra. Retrieved December 14, 2019, from https://www.ghanaweb.com/GhanaHomePage/NewsArchive/Urban-Sprawl-A-New-Epidemic-In-Accra-170196 
Traffic signal system to be centrally controlled. (2013, February 10). Retrieved December 4, 2019, from https://www.ghanaweb.com/GhanaHomePage/NewsArchive/Traffic-signal-system-to-be-centrally-controlled-264625

Smith, D. (2015, June 5). Death toll rises in Accra floods and petrol station fire. Retrieved December 2, 2019, from https://www.theguardian.com/world/2015/jun/05/death-toll-accra-floods-petrol-station-fire

African Development Bank (2016, September 29). Accra Urban Transport Project to address the challenges of a rapidly growing city. Retrieved November 29, 2019, from https://www.afdb.org/en/news-and-events/accra-urban-transportproject-to-address-the-challenges-of-a-rapidly-growing-city-16169

World Bank Group (2017, May). Enhancing Urban Resilience in the Greater Accra Metropolitan Area. Retrieved November 2019, from http://documents.worldbank.org/curated/en/949241495793834492/pdf/115296REPLACEMENT-PUBLIC-Accra-v5-highres-nocutmarks.pdf

Water \& Sanitation for the Urban Poor. (2018, April). Retrieved December 6, 2019, from https://www.wsup.com/wherewe-work/ghana/accra/

GH-GAMA Sanitation and Water Project. (2019, November 30). Retrieved December 8, 2019, from https://projects.worldbank.org/en/projects-operations/project-detail/P119063?lang=en 